\title{
Mushroom Poisoning with Symptoms of Pantherina Syndrome: A Case Report
}

\author{
Çağdaş Yıldırım', Gülhan Kurtoğlu Çelik', Güllü Ercan Haydar', Gül Pamukçu Günaydın', Yavuz Otal', Ayhan Özhasenekler² \\ 'Department of Emergency Medicine, Ankara Atatürk Training and Research Hospital Ankara, Turkey \\ ${ }^{2}$ Department of Emergency Medicine, Yıldırım Beyazıt Universitesi School of Medicine, Ankara, Turkey
}

\begin{abstract}
Introduction: The emergency department is the most common site of presentation for patients suffering from acute mushroom poisoning; therefore, emergency physicians should be familiar with it. We present a case of mushroom poisoning that was initially misdiagnosed as a cerebrovascular event.

Case Report: A 59-year-old male patient was referred to our emergency service from another hospital with the presumptive diagnosis of a cerebrovascular event. He had presented to the other hospital with complaints of a decrease in consciousness and hallucinations that started $2 \mathrm{~h}$ ago. Upon presentation he was unconscious. Family members stated that 30 min before his symptoms started, he had eaten mushrooms that he had collected himself from nature. Active charcoal was administered after gastric lavage. Diazepam was given for agitations. Fifteen hours after the ingestion of mushrooms, the patient was fully conscious. He was monitored for 36 hours and was discharged uneventfully.

Conclusion: Pantherina syndrome is a type of mushroom poisoning that has been classified as glutaminergic neurotoxic syndrome with early onset. Treatment is mainly supportive and should be guided by the patient's symptoms rather than by attempts at mushroom identification. Elimination of the mushroom from the gastrointestinal tract by gastric lavage and the administration of activated charcoal is recommended. Generally, the symptoms are mild and require no further treatment. Emergency physicians should consider mushroom poisoning in the evaluation of patients with acute onset delirium who may be intoxicated by natural substances.
\end{abstract}

Keywords: Amanita pantherina, mushroom poisoning, pantherina syndrome

Received: 03.09.2015 Accepted: 01.02.2016 Available Online Date: 14.06.2016

\section{Introduction}

There are about 500 species of mushrooms in nature, around 50-100 of which are known to be toxic to humans (1). Depending on the type of mushroom, adverse effects range from mild gastrointestinal (Gl) symptoms to major cytotoxic effects resulting in organ failure and death (1).

Mushroom poisoning occurs frequently in Turkey, especially in the beginning of summer, but also in spring and autumn (2, 3). The most frequent cause of poisoning is mistaking poisonous mushroom species for edible mushroom species.

Amanita pantherina is a kind of mushroom with hallucinogenic effects. It has a length of 8-9 cm and a cap size of 8-10 cm. It has a yellowish brown color and can grow in clusters of two or three. (4) Its appearance may be mistaken for that of non-poisonous species such as A. rubescens, A. spissa, or Macrolepiota procera (5).

Patient history is very important in the diagnosis of mushroom poisoning. In the most deadly mushroom intoxications, there is a $6 \mathrm{~h}$ or longer delay between symptom onset and mushroom consumption; therefore, the association of the symptoms with mushrooms may be difficult (1). Although identification of a characteristic toxidrome may make the diagnosis easier, the presenting symptoms and signs can be confused with other benign clinical syndromes (1). 
The emergency department is the most common site of presentation for patients suffering from acute mushroom poisoning (1); therefore, emergency physicians should be familiar with it. We present a case of mushroom poisoning that was initially misdiagnosed as a cerebrovascular event.

\section{Case Report}

A 59-year-old male patient was referred to our emergency service from another hospital with the presumptive diagnosis of cerebrovascular event. The patient had presented to the other hospital with complaints of a decrease in consciousness $2 \mathrm{~h}$ ago. According to the information obtained from the relatives of the patient, he was normal at the beginning of the day, but his level of consciousness decreased gradually after lunch and he then started to talk nonsense. Then, he became unconscious. The patient had a history of diabetes and hypertension. He was regularly on amlodipine, metformin, and insulin lispro.

Upon his arrival, he was unconscious, and his Glasgow Coma Scale (GCS) score was 9. His vital signs were as follows: blood pressure: 232/113 mmHg; heart rate: 90/min; and body temperature: $36.6^{\circ} \mathrm{C}$. His pupils were mydriatic. His four extremities were flexed in response to painful stimulant. An oropharyngeal airway was inserted to secure his airway initially. His blood glucose level drawn from the fingertip was $422 \mathrm{mg} / \mathrm{dL}$; he did not have acidosis but he had mild ketonuria. To improve hyperglycemia, $250 \mathrm{ml} / \mathrm{h}$ intravenous normal saline solution was administered. Brain computed tomography (CT) was obtained with the presumptive diagnosis of a cerebrovascular event. His CT revealed no abnormal findings that could be interpreted as bleeding or ischemia. His complete blood count, serum electrolytes, and liver and kidney function tests and urinalysis were normal, except his white blood cell count was $13.9 \mathrm{~K} / \mu \mathrm{L}$, venous blood glucose level was 344 mg/dL, and urine ketone was +1.

Family members stated that before losing consciousness, the patient called and told them that there was a tingling sensation in his arms and he had eaten the mushrooms that he collected from nature 30 min before symptom onset. Mushroom poisoning was considered, and a nasogastric catheter was inserted. Because the patient had a GCS score of 9, he was intubated to secure the airway. After gastric lavage, active coal was administered via a nasogastric catheter. Four hours later, following treatment, blood sugar was reduced to 201 $\mathrm{mg} / \mathrm{dl}$. About $10 \mathrm{~h}$ after eating mushrooms, the patient regained consciousness but he was agitated. Additionally, 5 mg IV diazepam was administered. Active coal was given again. The patient started to speak around $12 \mathrm{~h}$ after the ingestion of mushrooms. He had auditory hallucinations and was agitated again. Further 5 mg IV diazepam was readministered. Fifteen hours after the ingestion of mushrooms, the patient was fully conscious. He told that loss off sensation in his hands started 15 min after eating mushrooms, followed by visual hallucinations in $30 \mathrm{~min}$. He could not remember after that. He later recognized $A$. Pantherina in the pictures he was shown.

The patient was monitored for $36 \mathrm{~h}$ in the emergency service. His liver function tests, kidney function tests, and coagulation parameters did not yield any abnormality and he was discharged. Following discharge, the patient brought the rest of the mushrooms he collected to the emergency service where their pictures were taken (Figure 1).

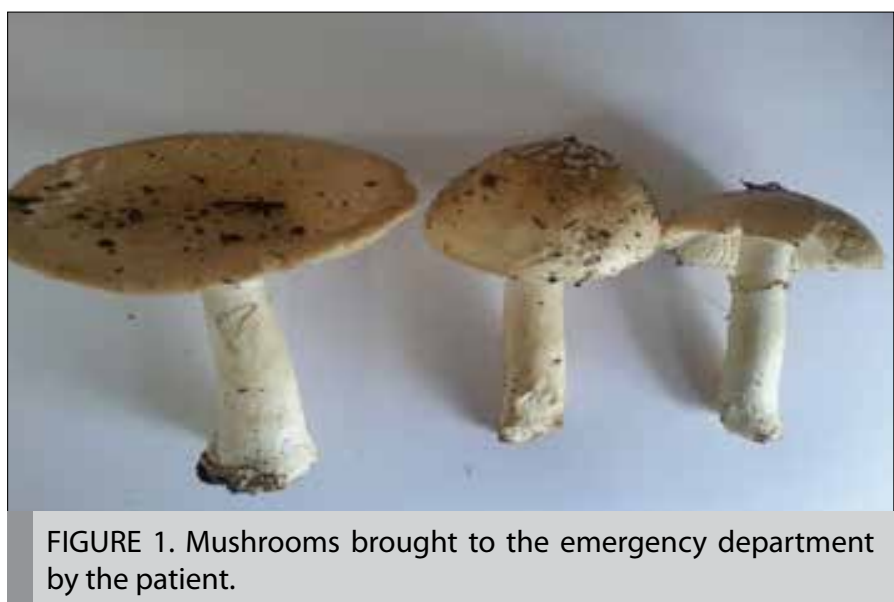

\section{Discussion}

Mushroom poisoning occurs among four main groups of individuals: young children who ingest mushrooms inadvertently, wildmushroom foragers, individuals attempting suicide or homicide, and individuals looking for a hallucinatory high (2). Our patient was poisoned with the mushrooms he collected from nature.

There are many described clinical syndromes of mushroom poisoning, and they are classified as early ( $<6 \mathrm{~h})$, late (6-24 h), and delayed ( $>1$ day) according to the onset time of symptoms. Pantherina syndrome has been classified as glutaminergic neurotoxic syndrome with early onset (6). Clinical symptoms have been reported in humans 30 min to $2 \mathrm{~h}$ after the ingestion of $A$. pantherina and can last up to $12 \mathrm{~h}(4,7)$. The symptoms of pantherina syndrome are nausea, hallucinations, delirium, muscular spasm, and deep sleep (5). Confusion, dizziness and tiredness, visual and auditory hypersensitivity, dryness of the mouth, mydriasis, hallucinations, vivid color perception, ataxia, and bradycardia have also been reported $(4,7)$. Coma is the final step of poisoning. In most cases, as little as one cap of mushrooms is sufficient for psychotropic effects (4). Central nervous system dysfunctions and neurologic symptoms primarily characterize this poisoning (4). Symptoms may fluctuate between lethargy and hyperkinetic behavior and from obtundation to delirium. Seizures are rarely encountered in adults (7). In our case, symptoms started about 30 min after the ingestion of mushrooms, and the main symptoms were a decrease of consciousness and hallucinations.

Active toxins in the A. pantherina causing the symptoms of poisoning are neurotoxic isoxasoles ibotenic acid and muscimol $(4,7)$. Ibotenic acid stimulates NMDA receptors, and muscimol stimulates GABA receptors (7). Cooking does not influence the level of toxins (4). Our patient ate the mushrooms cooked.

A. muscaria can also cause similar symptoms. Both mushrooms can cause confusion, agitation, hallucinations, convulsions, somnolence, coma, vomiting, and diarrhea. A. muscaria contains more excitatory ibotenic acid, whereas A. pantherina contains more inhibitory muscimol. Therefore, A. muscaria poisoning more often results in confusion and agitation, whereas A. pantherina poisoning causes coma more often (8). Our patient was brought in coma. 
Treatment is mainly supportive and should be guided by the patient's symptoms rather than by attempts at mushroom identification (2). Elimination of the mushroom from the gastrointestinal tract by gastric lavage and administration of activated charcoal is recommended (4). Generally, the symptoms are mild and require no further treatment (7). If seizures occur, they respond to conventional therapy with benzodiazepines and other anticonvulsants. Benzodiazepines should also be used for agitation and delirium. Atropine should be reserved for patients with symptomatic bradycardia (7). Severe poisonings may result in coma, necessitating intubation (7). Intensive care is recommended for those patients. In our case, benzodiazepines were used for the treatment of agitations.

Prognosis of A. pantherina poisoning is generally good, although it may rarely be lethal (4). In most cases, recovery is virtually complete after $24 \mathrm{~h}$ without noticeable after-effects (4). Our patient recovered in 15 h. Severe neuron and even brain lesions could be anticipated in cases of recurrent consumption (4).

Prevention and education are essential elements of care. Remembering the toxidromes is more important than recalling specifics about individual mushrooms (1). There are thousands of species of mushrooms. Although identifying the exact mushroom species might be helpful, it is not essential for effective management. Nonlethal mushrooms generally cause symptoms within 6 h, but because the patients often ingest more than one type of mushroom, the symptom onset within $6 \mathrm{~h}$ does not rule out a co-ingestion of a potentially dangerous variety in the food $(1,5,7)$. If there are any mushrooms remaining, it may be helpful for identification. Our patient had brought the remaining mushrooms for diagnostic purposes, and the emergency physician evaluated the mushrooms, but unfortunately they were not sent to a mycologist for analysis and confirmation.

Although a history of mushroom ingestion and its related toxidrome is helpful in the diagnosis, the symptoms can be variable and can easily be confused with benign clinical syndromes, such as gastroenteritis (3). In a retrospective study conducted by Eren et al. (2), the most common symptoms due to mushroom poisoning were reported to be nausea, vomiting, fatigue, abdominal pain, dizziness, diarrhea, headache, and loss of consciousness.

\section{Conclusion}

Emergency physicians should consider mushroom poisoning in the evaluation of all patients who may be intoxicated by natural sub- stances. A toxidromic classification of mushroom poisoning is recommended to guide clinicians in making earlier diagnoses, especially for cases that may need advanced care, such as liver or kidney transplantation (6).

Patient consent was not obtained because there is no information or picture of the patient in the manuscript that will allow the reader to recognize the patient.

Informed Consent: Written informed consent was not obtained from patient who participated in this case.

Peer-review: Externally peer-reviewed.

Author contributions: Concept - Ç.Y., G.K.Ç., G.P.G.; Design - Ç.Y., G.P.G., Y.O.; Supervision - A.Ö., G.P.G., G.K.Ç.; Resource - Ç.Y., G.E.H., Y.O.; Materials - G.E.H., Y.O., A.Ö.; Data Collection \&/or Processing - Ç.Y., G.K.Ç., Y.O.; Analysis \&/or Interpretation - G.P.G., Ç.Y., A.Ö.; Literature Search - G.P.G., C..Y., G.K.Ç.; Writing - Ç.Y., G.P.G., G.K.Ç.; Critical Reviews - G.P.G., A.Ö., G.K.Ç.

Conflict of Interest: The authors declared no conflict of interest.

Financial Disclosure: The authors declared that this study has received no financial support.

\section{References}

1. Berger KJ, Guss DA. Mycotoxins revisited: Part I. J Emerg Med 2005; 28: 53-62. [CrossRef]

2. Eren SH, Demirel Y, Uğurlu S, Korkmaz I, Aktaş C, Güven FM. Mushroom poisoning: retrospective analysis of 294 cases. Clinics (Sao Paulo) 2010; 65: 491-6. [CrossRef]

3. Yardan T, Baydin A, Eden AO, Akdemir HU, Aygun D, Acar E et al. Wild mushroom poisonings in the Middle Black Sea region in Turkey: analyses of 6 years. Hum Exp Toxicolo 2010; 29: 767-71. [CrossRef]

4. Michelot D, Melendez-Howel LM. Amanita muscaria: chemistry, biology, toxicology, and ethnomycology. Mycol Res 2003; 107: 131-46. [CrossRef]

5. Satora L, Pach D, Ciszowski K, Winnik L. Panther cap Amanita pantherina poisoning case report and review. Toxicon 2006; 47: 605-7. [CrossRef]

6. Diaz JH. Syndromic diagnosis and management of confirmed mushroom poisonings. Crit Care Med 2005; 33: 427-36. [CrossRef]

7. Berger KJ, Guss DA. Mycotoxins revisited: Part II. J Emerg Med 2005; 28 : 175-83. [CrossRef]

8. Vendramin A, Brvar M. Amanita muscaria and Amanita pantherina poisoning: two syndromes. Toxicon 2014; 90: 269-72. [CrossRef] 